\section{Color Image Analysis In Optical Microscopy}

Sylvain Laroche

CLEMEX Technologies, Longueuil, Canada

Most of the applications in microscopic image analysis are still solved by a heuristic method that focuses on the specificity of each problem. This is particularly tue for applications involving color images. Because of this, the design of robust algorithms has remained time consuming and is usually performed by an image analysis expert with many years of experience. How then can the knowledge of the expert be transferred to the less specialized microscopist? How can the specificity of a typical application be related to general color image analysis tools?

Only a general approach can work towards decreasing the heuristic aspect of the design phase and help the common user perform image analysis without the assistance of an expert. To illustrate such an approach, two typical applications, one in material science, and the other in the biomedical field are presented:

Segmentation of aluminum grains seen under polarized light

Detection of DNA-multi-probes in fluorescence imaging in-situ hybridization (FISH).

Although these applications are very different in nature, the image analysis framework is the same. The implementation of such a framework in a fully integrated image analysis system is also discussed.

Detecting and measuring objects of interest described by specific colors, shape and contrast requires tools that fall within the following categories:
1) Color acquisition and color space conversion
2) Color Filtering
3) Color Segmentation

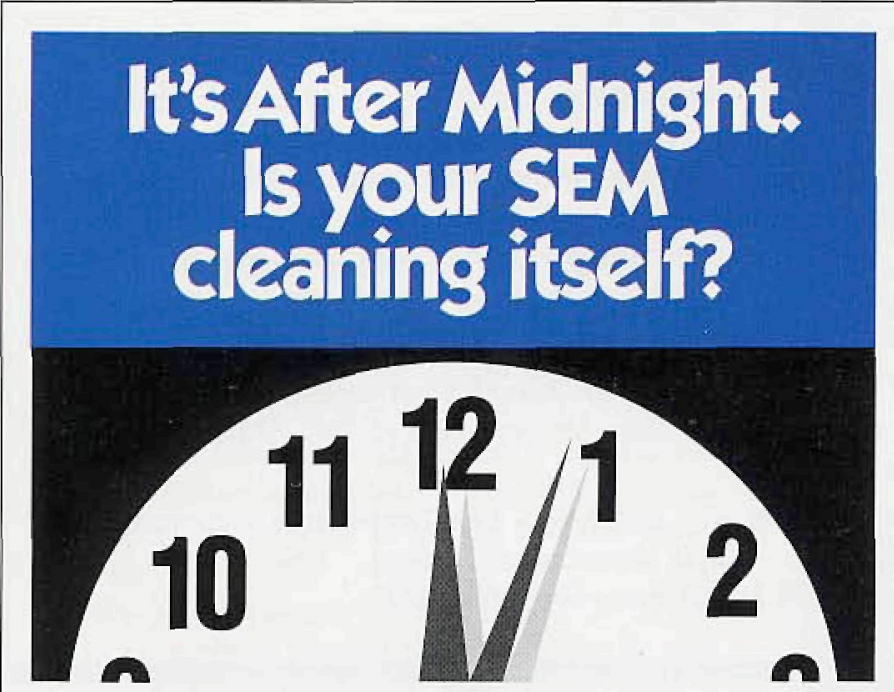

It would be if you had the SEM-CLEAN ${ }^{\top M}$ anti-contamination system. Overnight, the inexpensive SEM-CLEAN system uses a Nitrogen purge to clean your electron microscope. Its active cleaning action removes oils and other adsorbed contaminants from the walls of the chamber and carries them to the roughing pump. Result? Significant reductions in, even elimination of, sample-contamination, raster burns and oil buildup on EDS windows. Over 50 satisfied SEM-CLEAN users are our best references. For a list and complete details, call or write today.

\section{SEM-CLEAN'" Stops the Oil}

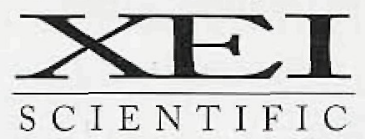

3124 Wessex Way, Redwood City, CA, U.S.A 94061 415-369-0133 - Fax 415-363-1659
4) Edge Detection

5) Measurement and mapping over the full sample

Several color models are available to process color images. The well: known RGB format is very common for display purposes. However, it is inadequate for image analysis since the linear combination of the three components is not easily deduced for expressing a region with similar colors? Consequently, the process of color segmentation is very difficult. An alternate color space, HSI (Hue, Saturation, Intensity), is more suitable and is closer to the way color is expressed in everyday life. Because video cameras deliver an RGB signal, a conversion from RGB to HSI is required. Since display systems areo restricted to RGB output, representing an $\mathrm{HSI}$ image is not a simple issue $\frac{\bar{\sigma}}{\bar{n}}$ Specifically, a further conversion back to the RGB space becomes necessary. $\frac{\overrightarrow{0}}{2}$

Other factors also mitigate the acquisition process. For example, uneverp lighting at the microscopic level must be compensated by a special form op shading correction performed in the HSI space. In FISH applications, low-ligh ${ }^{\circ}$ fluorescence imaging also demands a special acquisition mode becausę standard CCD cameras are not sensitive enough to capture as many photons as:required. Consequently, increasing the exposure time of the CCD element is often necessary to deal with such conditions.

The raw HSI image contains objects of interest as well as artifacts background structures, etc. To facilitate the segmentation process, color filtering can be used to enhance the objects of interest while discarding other features? Mathematical Morphology has proven to be a very powerful approach to segment and filter gray-tone and binary images. However, common operations such as erosion, dilation, opening and closing do not have clear counterparts in color imaging. Such transforms can nonetheless be implemented into the HSI space by performing morphological operations on the intensity component and propagating the Hue-Saturation pair over the neighborhood of the structuring element. The generalization of Mathematical Morphology to color image processing has lead to the creation of several powerful non-linear filters.

After proper filtration, objects having specific colors are detected by segmentation in the HSI color space. Low and high limits for hue, saturation and intensity can easily be defined as long as the HSI space is presented to the user in a relevant manner. An HSI color histogram can also be used to facilitate the color thresholding process.

Performing color thresholding immediately, even after filtering, is not always the most efficient approach however. For example, when adjacent color structures are in contact, color segmentation can be more easily obtained by detecting the color boundary. In such cases, a color edge gradient can be performed. The resulting noisy image is then further processed by applying a watershed operation to yield a clear boundary structure despite the poor local contrast of the gradient image. This image can then be segmented by thresholding.

Filtering is usually required to clean the binary image obtained by the color segmentation before measurements and shape factors can be computed for the objects of interest. Once the filtering is complete, object measurements can be performed to yield a final classification. The amount of data generated can be somewhat overwhelming, particularly if data was gathered over several fields. This can make the review and validation process tedious if the proper tools are unavailable. One effective approach is to map the results over the full sample and superimpose the map over the original image. The operator then inspects the map to validate the results.

1. Laroche $S_{\text {., Forget }}$., Grain Sizing of Anodized Aluminum by Color Image Analysis, MC95, International Metallography Conference, May 1995.

2. Serra J., Image Analysis and Mathematical Morphology, Academic Press, 1982. $610 \mathrm{p}$.

3. Breen E.J., Regression methods for automated colour image classification and thresholding, Journal of Microscopy, vol. 174. Pt 1, April 1994, 23-30.

Reprinted from the proceedings of Microscopy \& Microanalysis 1996.

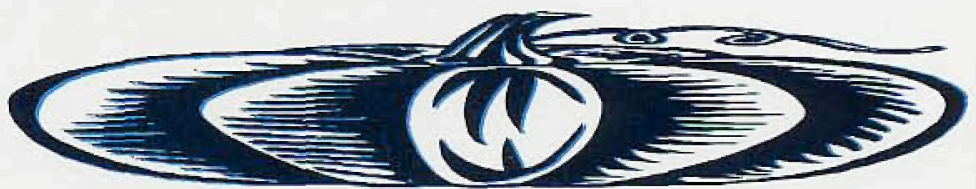




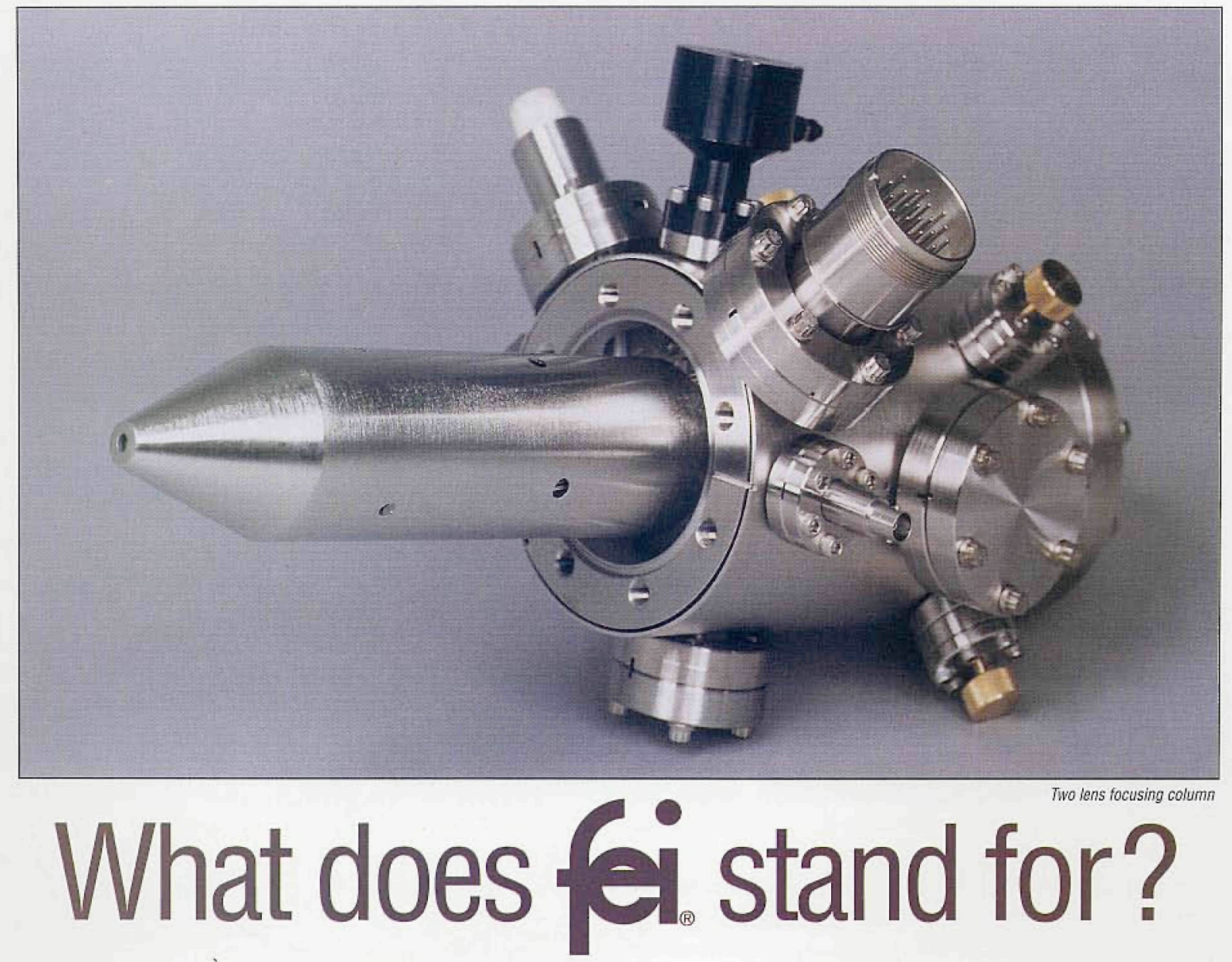

Our compact, UHV, field emission columns are used by researchers world wide. Innovative electrostatic optics and dedicated electronics allow you to integrate a high current density electron or ion column into most vacuum systems. FEI also supplies researchers with other specialized products...

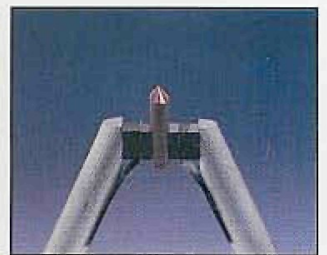

$\mathrm{LaB}_{6}$ an $\mathrm{CeB}_{6}$ Cathodes

FEl's Mini-Vogel Mount, the first universally compatible long-life, high-stability LaB6 cathode, provides excellent performance and the best cost-per-use value for installation into your EM systems.

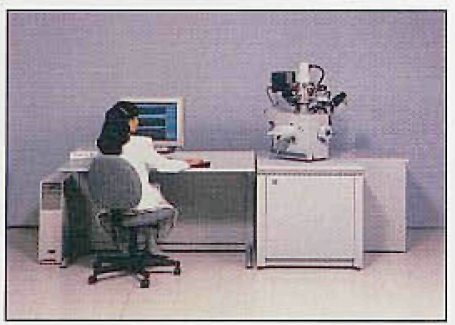

\section{FIB Workstations}

Focused ion beam micromilling workstations range from the 8"-wafer compatible model to the economical 2" small sample model ideal for semiconductor, biological, TEM, and MEMS specimens.

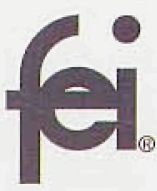

FEl Company

7451 NE Evergreen Parkway

Hillsboro, OR 97124-5830

(503) 640-7500 Fax (503) 640-7509

email:rsk@feico.com

Now, when you think of FEI, you'll know we are the Specialists in Field Electron and Ion Technology. 